\title{
A beta cell-specific knockout of hormone-sensitive lipase in mice results in hyperglycaemia and disruption of exocytosis
}

\author{
M. Fex • G. Haemmerle • N. Wierup • \\ M. Dekker-Nitert • M. Rehn • M. Ristow $\cdot$ R. Zechner • \\ F. Sundler • C. Holm • L. Eliasson • H. Mulder
}

Received: 7 July 2008 / Accepted: 25 September 2008 / Published online: 21 November 2008

(C) Springer-Verlag 2008

\begin{abstract}
Aims/hypothesis The enzyme hormone-sensitive lipase (HSL) is produced and is active in pancreatic beta cells. Because lipids are known to play a crucial role in normal control of insulin release and in the deterioration of beta cell function, as observed in type 2 diabetes, actions of HSL in beta cells may be critical. This notion has been addressed in different lines of HSL knockout mice with contradictory results.

Methods To resolve this, we created a transgenic mouse lacking HSL specifically in beta cells, and characterised this
\end{abstract}

Electronic supplementary material The online version of this article (doi:10.1007/s00125-008-1191-9) contains supplementary material, which is available to authorised users.

M. Fex $(\bowtie) \cdot$ M. Dekker-Nitert $\cdot$ L. Eliasson $\cdot$ H. Mulder

Department of Clinical Sciences,

Clinical Research Centre (CRC),

Malmö University Hospital (UMAS),

Malmö, Sweden

e-mail: malin.fex@med.lu.se

N. Wierup $\cdot$ M. Rehn $\cdot$ F. Sundler $\cdot$ C. Holm

Department of Experimental Medical Science,

Lund University,

Lund, Sweden

M. Fex $\cdot$ N. Wierup $\cdot$ M. Dekker-Nitert $\cdot$ F. Sundler •

C. Holm $\cdot$ L. Eliasson $\cdot$ H. Mulder

Lund University Diabetes Centre,

Lund, Sweden

G. Haemmerle $\cdot$ R. Zechner

Institute of Molecular Biosciences, University of Graz,

Graz, Austria

M. Ristow

Department of Human Nutrition, University of Jena,

Jena, Germany model with regard to glucose metabolism and insulin secretion, using both in vivo and in vitro methods.

Results We found that fasting basal plasma glucose levels were significantly elevated in mice lacking HSL in beta cells. An IVGTT at 12 weeks revealed a blunting of the initial insulin response to glucose with delayed elimination of the sugar. Additionally, arginine-stimulated insulin secretion was markedly diminished in vivo. Investigation of the exocytotic response in single HSL-deficient beta cells showed an impaired response to depolarisation of the plasma membrane. Beta cell mass and islet insulin content were increased, suggesting a compensatory mechanism, by which beta cells lacking HSL strive to maintain normoglycaemia. Conclusions/interpretation Based on these results, we suggest that HSL, which is located in close proximity of the secretory granules, may serve as provider of a lipidderived signal essential for normal insulin secretion.

Keywords Beta cell mass - Insulin secretion . Lipid coupling factor Membrane capacitance

\begin{tabular}{|c|c|}
\hline Abbreviations & \\
\hline AIR & acute insulin response \\
\hline ATGL & adipocyte triacylglycerol lipase \\
\hline beta HSL KO & beta cell-specific knockout of HSL \\
\hline DEXA & dual-energy X-ray absorptiometry \\
\hline HSL & hormone-sensitive lipase \\
\hline ITT & insulin tolerance test \\
\hline$\alpha-\mathrm{KIC}$ & $\alpha$-ketoisocaproic acid \\
\hline $\mathrm{KO}$ & knockout \\
\hline LC-CoAs & long-chain acyl-CoAs \\
\hline PKA & protein kinase A \\
\hline RIP2 & rat insulin 2 promoter \\
\hline WT & wild-type \\
\hline
\end{tabular}




\section{Introduction}

It is now widely accepted that failure of insulin secretion is the pre-eminent contributor to type 2 diabetes. Lipid signalling is regarded by many as a critical and possibly pathogenetic factor in the control of insulin secretion [1]. Several models describing intracellular and extracellular pathways for this control have been proposed. Lipolysis, i.e. the breakdown of triacylglycerol to NEFA and glycerol, in beta cells, has emerged as an important but controversial process. Hormone-sensitive lipase (HSL) controls lipolysis in adipocytes [2]; it has a broad substrate specificity, hydrolysing tri-, di- and monoacylglycerols, as well as cholesteryl and retinyl esters [3]. The activity of HSL is controlled by hormones via changes in intracellular cAMP levels and the consequent phosphorylation of protein kinase A (PKA) [4].

We have previously shown that HSL is active in pancreatic beta cells [5]. Here, HSL may generate lipid coupling signals for insulin secretion via lipolysis, including long-chain acyl-CoAs (LC-CoAs) and diacylglycerol [1, 6-9]. To this end, LC-CoAs have been shown to stimulate insulin secretion, possibly via the activation of protein kinase $\mathrm{C}$ [10], direct interaction with the exocytotic machinery [11] or via activation of the ATP-sensitive $\mathrm{K}^{+}$ channel [12]. The critical role of lipolysis has been further highlighted by studies using lipase inhibitors: thus orlistat, a pan-lipase inhibitor, blocks lipolysis and insulin secretion in rat islets [13], while a selective inhibitor of HSL has been shown to inhibit insulin secretion from rat islets [14]. Moreover, a positive correlation between lipolysis and insulin secretion exists in INS-1 cells and mouse islets [15]. Hence, loss of HSL from beta cells could block mobilisation of lipids from stored triacylglycerol and abrogate lipid signalling. With time, triacylglycerol could accumulate within the pancreatic beta cell. The detrimental effect of this could be dual. First, abolished lipid signalling could impair insulin secretion; second, the excess of stored lipid in the beta cell could arguably lead to cellular dysfunction, i.e. lipotoxicity, and ultimately type 2 diabetes $[16,17]$.

Four independent lines of HSL knockout (KO) mice have been reported [18-21]. While HSL KO mice are lean, all lines exhibit male infertility $[18,22,23]$. In half of the HSL KO lines, some indication of insulin resistance exists, the exception being the Ishibashi line, where fasting plasma glucose levels are either unchanged or decreased, while insulin levels are unaltered [24], and the Zechner line, which exhibits increased insulin sensitivity in liver [20]. Increased hepatic insulin sensitivity was later reproduced in the Mitchell HSL KO line [25]. Euglycaemic-hyperinsulinaemic clamps have not clarified the issue: our HSL KO line displayed impaired hepatic insulin response [21], while that of Mitchell showed an exaggerated response [25]. In terms of insulin secretion, our line of HSL KO mice responds vigorously to glucose and arginine in vivo [21], and to several other secretagogues in vitro [26]. In contrast, Prentki's group reported perturbed insulin secretion in vivo and in vitro [27, 28] in the line produced by Mitchell [19], a phenotype evident in male mice and pronounced upon fasting. Female mice, however, of this HSL KO line exhibit increased glucose-stimulated insulin secretion in islets isolated from fed and fasted animals [28]. The discrepancies in and between the genetically targeted mice may be attributed to different genetic backgrounds, redundancy and/or the compensatory actions of other lipases. Indeed, a novel lipase, adipocyte triacylglycerol lipase (ATGL), has been discovered in HSL KO mice [29, 30], and was found to be expressed also in beta cells and islets [30].

To elucidate a role for HSL in insulin secretion, we created a genetic model for HSL-deficiency in beta cells, which should be less prone to problems inherent in the preexisting global HSL KO lines. A beta cell-specific KO of HSL (beta HSL KO) was created by Cre-mediated recombination (rat insulin 2 promoter [RIP2] Cre mouse) [31-33] in the beta cells of a transgenic mouse with LoxP sites flanking exons 2 to 7 in $H s l$ (also known as Lipe) [20]. In the present study, we show that a beta cell-specific loss of HSL results in hyperglycaemia due to impaired insulin secretion. Our data support the notion that the lipase plays a critical role in beta cell stimulus-secretion coupling.

\section{Methods}

Reagents and ethical approval All reagents were from Sigma (St Louis, MO, USA) unless otherwise stated. The studies were approved by The Regional Animal Ethics Committee in Lund, Sweden.

Animals and breeding strategies An SV129/C57BL/6J mouse line carrying a mutated $H s l$ allele, in which exons 2 to 7 in the $H s l$ gene were flanked by LoxP sites [20], was crossed with a line $(\mathrm{C} 57 \mathrm{BL} / 6 \mathrm{~J})$ expressing the $\mathrm{CRE}$ recombinase under control of the RIP2 [31, 32]. Breeding pairs of the genotype $\mathrm{Hs}_{s} \mathrm{H}^{/-} / \mathrm{Cre}^{+/-}$and $\mathrm{Hs}_{s} \mathrm{l}^{++}$were used to produce the offspring $\mathrm{Hsl}^{+/+}, \mathrm{Hsl}^{+/-}, \mathrm{Hs}_{s}^{+/+} / \mathrm{Cre}^{+/-}$and $\mathrm{Hsl}^{+/-}$ $\mathrm{Cre}^{+/-} . \mathrm{Hsl}^{+/+}$was used as control (wild-type [WT]/Lox) and the $\mathrm{Hsl}^{+/+} / \mathrm{Cre}^{+/-}$genotype were the beta HSL KO. Female mice were used in all experiments unless otherwise stated.

Tissue isolation and RT-PCR mRNA was prepared either from frozen adipose tissue or fresh islets (mRNA Easy Plus mini kit; Qiagen, Hilden, Germany) from 5-week-old beta HSL KO and WT/Lox mice. cDNA was obtained by reverse transcription. PCR with the following primers was 
performed: (1) 5'-CATGCACCTAGTGCCATCCTTC-3'; (2) 5'-CTCACTGAGGCCTGTCTCGT TG-3'; and (3) 5'TACCGAGCCATCTTCCTAGTCC- $3^{\prime}$. Primers 1 and 2 were used to detect the full-length HSL construct (390 bp) and primers 1 and 3 the truncated HSL construct (250 bp), which were separated on a $1 \%$ agarose gel.

Blood sampling and analysis Blood was collected by retroorbital sampling. Glucose was determined in plasma by Infinity Glucose Ox (TR 1521-125; Thermo Electron, Melbourne, VIC, Australia). Insulin, leptin and adiponectin in plasma were measured by radioimmunoassay (Linco Research, St Charles, MO, USA).

Tolerance tests Intravenous glucose tolerance, arginine tolerance and insulin tolerance tests were performed as previously described [21] and responses calculated as AUC using the trapezoid model. In all in vivo investigations, plasma glucose and hormone levels were determined in retro-orbital blood samples collected at the time-points indicated. For fasting samples, mice were deprived of food at 23:00 hours and samples were taken from anaesthetised mice at 7:00 hours.

Insulin secretion in vitro Islets were isolated and insulin secretion experiments were performed as described [26]. Assay buffer samples were removed for measurement of insulin by ELISA (Novo Nordisk, Copenhagen, Denmark).

Capacitance and ion current measurements in single beta cells Single islet cells were dissociated, using a $\mathrm{Ca}^{2+}$-free solution and cultured as described [34]. Whole-cell currents and exocytosis were measured in standard whole-cell configuration of the patch-clamp technique as described [34]. Exocytosis was detected as changes in membrane capacitance, using a software-based lock-in application of the amplifier (Pulse software version 8.50; HEKA Electronik, Lambrecht, Göttingen, German). Experiments were conducted at 32 to $34^{\circ} \mathrm{C}$.

Immunocytochemistry and measurement of beta cell mass Immunocytochemistry and measurement of beta cell mass were performed as described [35]. Digitised images of all islets in three sections per animal were analysed. Islet areas were measured and the mean area of insulin staining was calculated using Image Pro Plus Software (Media Cybernetics,Bethesda, MD, USA); the identity of the sections was unknown to the investigator.

Oil Red O staining and optical density analysis Oil Red O (Fluka Chemie, Buchs, Switzerland) was prepared. Sections $(10 \mu \mathrm{m})$ from frozen pancreas were cut in a cryostat, thawed, air-dried and fixed [35]. As an index of neutral lipid content that would reflect triacylglycerol content, optical density was measured in four randomly chosen slides from the KO mice and three slides from controls.

Mouse body scan A whole-body scan (dual-energy X-ray absorptiometry [DEXA]) of mice as described [36] was used to determine fat distribution as percentage body fat.

Quantitative real-time PCR Quantitative real-time PCR was performed on islet beta HSL KO and WT/Lox cDNA. A custom-made Taqman gene expression assay (Applied Biosystems, Foster City, CA, USA) for mouse Atgl (also known as Pnpla2) was employed (NM_025802). The cycle threshold values obtained by the real-time PCR reaction (7900HT Thermal Cycler; Applied Biosystems) were normalised to the values for mouse beta actin and human 18S RNA (Hs999901.s1).

Statistical analysis Values are given as means \pm SEM. Data were analysed with a two-tailed unpaired $t$ test unless otherwise stated. In all tolerance tests, AUC was calculated and compared with a two-tailed $t$ test. Data analysis of realtime PCR was performed with the Kruskal-Wallis test with Dunn's test post hoc. $p$ values of $<0.05$ were considered significant.

\section{Results}

Cre-mediated recombination in beta cells abrogates Hsl expression in islets PCR products from RT-PCR reactions on adipose tissue and islet cDNA from 5-week-old beta HSL KO and WT/Lox mice are shown in Fig. 1. cDNA from islet beta HSL KO mice contained the truncated HSL construct $(250 \mathrm{bp})$, indicating that recombination has occurred. Adipose tissue cDNA from beta HSL KO and WT/Lox, and islets from WT/Lox contained the full length construct (390 bp). Thus, recombination in beta cells was efficient, having created a specific knockout of HSL in beta cells.

Glucose disposal in beta HSL KO mice is delayed due to a blunted acute insulin response We found that basal plasma glucose levels were significantly elevated in 12-week-old beta HSL KO mice (Fig. 2a). An IVGTT in 12-week-old fasted mice $(8 \mathrm{~h})$ revealed delayed glucose clearance in beta HSL KO mice (Fig. 2b; for incremental AUC, see Table 1). The initial insulin response was severely blunted in beta HSL KO mice (Fig. 2c). The acute insulin response (AIR) to glucose (AIR Glucose $_{\text {) was }} 840 \pm 190$ versus $175 \pm 48$ pmol $1^{-1} \min ^{-1}$ in WT/Lox versus beta HSL KO mice, respectively $(p<0.001)$ (Table 1$)$. The differences in $\mathrm{AIR}_{\text {Glucose }}$ suggest a selective loss of the first phase of insulin secretion 


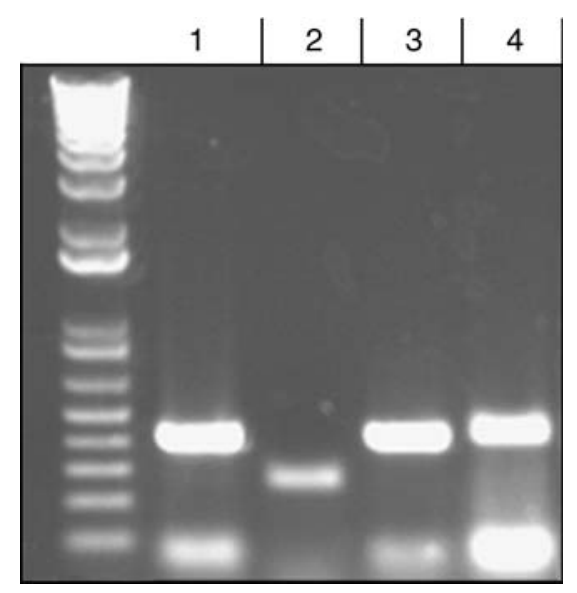

Fig. 1 PCR products from islets and adipose tissue. cDNA isolated from 5-week-old WT/Lox and beta HSL KO mice. Lane 1: PCR product from WT/Lox islets yielding a $390 \mathrm{bp}$ band. Lane 2: PCR product from beta HSL KO islets yielding a $250 \mathrm{bp}$ band, consistent with a truncated HSL transcript. Lanes 3 and 4 show adipose (adi) tissue controls from WT/Lox and beta HSL KO mice, and contain only the full-length transcript (390 bp)

in the beta HSL KO mouse. An IVGTT was also performed in fed mice; here, glucose clearance was similar in both genotypes. However, the perturbed insulin response was still evident (Electronic supplementary material [ESM] Fig. 1a, b). Thus, mice lacking HSL in beta cells exhibited basal hyperglycaemia, decreased glucose elimination and a blunted initial insulin response.

Arginine reveals an impaired secretory capacity in beta HSL KO mice Beta HSL KO mice were challenged with arginine to determine the maximal stimulatory response. Beta HSL KO mice aged 12 weeks responded to arginine with a reduced insulin response (Fig. 3a; for AIR $_{\text {Arginine }}$ and AUC, see Table 1). Interestingly, arginine-stimulated glucagon secretion was also significantly reduced in beta HSL KO mice (Fig. 3b).

Insulin tolerance test in beta HSL KO mice Glucose clearance during the IVGTT was not significantly different between the two genotypes in fed animals despite impaired early insulin secretion, possibly indicating a compensatory increase in insulin sensitivity in the beta HSL KO animals. Therefore, an insulin tolerance test (ITT) was performed. Glucose disposal appeared to be slightly more efficient in beta HSL KO mice than in WT/Lox mice (Fig. 3c). This suggests enhanced peripheral sensitivity to insulin, which was corroborated by a significant increase in the $\Delta$-value at $T_{0}-T_{15}$ in beta HSL KO mice. However, calculation of the inverse $\mathrm{AUC}_{\mathrm{ITT}}$, another index of insulin sensitivity, revealed that glucose disposal upon insulin injection was actually impaired in beta HSL KO mice. These ambiguities may be due to the fact that basal plasma glucose was elevated in the beta HSL KO mice, calling for caution when interpreting the data (for $\Delta$ values and $\mathrm{AUC}_{\mathrm{ITT}}^{-1}$, see Table 1).

Insulin secretion from isolated islets revealed a loss of the initial insulin response Because the IVGTT suggested a selective loss of first-phase insulin secretion, we initially performed a short static incubation (10 $\mathrm{min})$ to assess early insulin secretion in vitro. Islets from WT/Lox mice responded with a 2.3-fold increase in insulin secretion when glucose levels were raised, while islets from beta HSL KO mice failed to respond (Fig. 4). The same pattern was observed when islets were stimulated with 2.8 or $16.7 \mathrm{mmol} / \mathrm{l}$ glucose and $70 \mathrm{mmol} / \mathrm{K} \mathrm{KCl}$. In contrast, in a $1 \mathrm{~h}$ static incubation, no significant differences in insulin secretion were detected between the genotypes upon

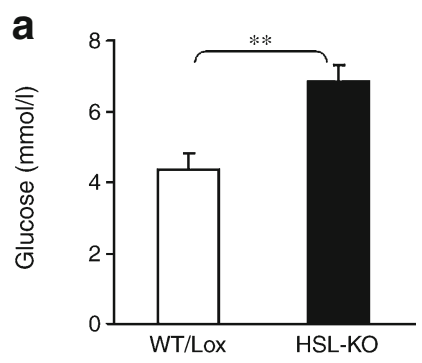

b
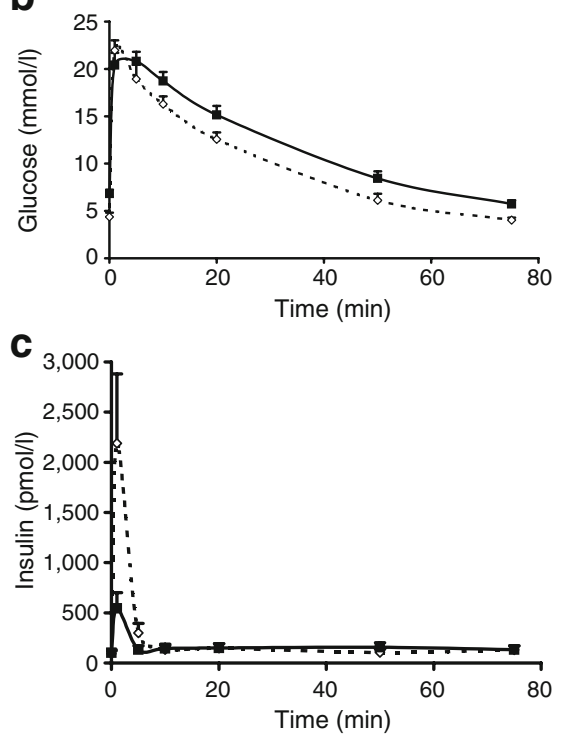

Fig. 2 Plasma glucose and IVGTTs. a Basal plasma glucose was elevated in 12-week-old beta HSL KO mice (black bar). WT/Lox mice (white bar) had normal basal plasma glucose. IVGTT (b) showed retarded glucose clearance in beta HSL KO mice (black squares) compared with WT/Lox mice (white diamonds). Insulin levels from IVGTT (c) were significantly reduced in plasma from beta HSL KO mice (black squares) in comparison with WT/Lox controls (white diamonds). For $\mathrm{AIR}_{\mathrm{Glucose}}$ and AUCs for glucose and insulin, see Table 1. Values are mean \pm SEM for two independent experiments where the number of animals in $\mathrm{KO}$ and control groups was $12 . * * p<0.01$ 
Table 1 AIR for IVGTT, insulin content, beta cell mass and body fat distribution in beta HSL KO mice

\begin{tabular}{|c|c|c|}
\hline Variables & WT/Lox & Beta HSL KO \\
\hline $\operatorname{AIR}_{\text {Arginine }}\left(\mathrm{pmol} \mathrm{l}^{-1} \min ^{-1}\right)$ & $1329 \pm 65$ & $339 \pm 85^{* * *}$ \\
\hline $\operatorname{AIR}_{\text {Glucose }}\left(\mathrm{pmol} 1^{-1} \min ^{-1}\right)$ & $840 \pm 190$ & $175 \pm 48^{* * *}$ \\
\hline$\Delta \operatorname{ITT}\left(T_{0}-T_{15}\right)^{\mathrm{a}}$ & $0.12 \pm 0.08$ & $0.3 \pm 0.13^{*}$ \\
\hline Inc $\mathrm{AUC}_{\text {Glucose }}\left(\mathrm{mmol} \mathrm{1} \mathrm{1}^{-1} \min ^{-1}\right)^{\mathrm{b}}$ & $347 \pm 62$ & $412 \pm 66^{* *}$ \\
\hline $\mathrm{AUC}_{\text {Insulin }}\left(\mathrm{pmol}^{-1} \min ^{-1}\right)^{\mathrm{b}}$ & $15300 \pm 755$ & $12073 \pm 1220^{*}$ \\
\hline $\mathrm{AUC}_{\text {Glucagon }}\left(\mathrm{ng} 1^{-1} \min ^{-1}\right)^{\mathrm{b}}$ & $5592.28 \pm 1411$ & $3905.37 \pm 1425^{*}$ \\
\hline Islet insulin content $(\mathrm{pmol} / \text { islet })^{\mathrm{c}}$ & $3.7 \pm 0.9$ & $8.7 \pm 1.2 * * *$ \\
\hline Beta cell mass $\left(\mu \mathrm{m}^{2}\right)^{\mathrm{d}}$ & $3.4 \pm 2.4$ & $8.8 \pm 6.8^{* *}$ \\
\hline Adiponectin $(\mu \mathrm{g} / \mathrm{ml})^{\mathrm{e}}$ & $32.7 \pm 3.5$ & $30.2 \pm 4.5$ \\
\hline Leptin $(\mathrm{ng} / \mathrm{ml})^{\mathrm{e}}$ & $3.5 \pm 0.4$ & $11.5 \pm 2.8^{*}$ \\
\hline Body fat $(\%)^{f}$ & $13.7 \pm 1.9$ & $18.9 \pm 2.9^{* *}$ \\
\hline
\end{tabular}

All values are presented as means \pm SEM

${ }^{*} p<0.05,{ }^{* *} p<0.01, * * * p<0.001$

${ }^{\mathrm{a}} \Delta$ Values (difference between $T_{0}-T_{15}$ ) for ITT and the inverse $\mathrm{AUC}_{\mathrm{ITT}}$

${ }^{\mathrm{b}}$ AUC for tolerance tests as well as the incremental AUC (Inc AUC) for glucose for WT/Lox and beta HSL KO animals respectively

${ }^{\mathrm{c}}$ Total islet insulin content in islets from 12-week-old beta HSL KO mice and WT/Lox littermates $(n=200$ islets from five mice of each genotype)

${ }^{\mathrm{d}}$ Assessed by morphometrical analysis in beta HSL KO and WT/Lox islets ( $n=7$ and 8 mice/genotype, respectively)

${ }^{\mathrm{e}}$ From 12-week-old beta HSL KO mice compared with WT/Lox mice $(n=10)$

${ }^{\mathrm{f}}$ Body fat accumulation (\%) in beta HSL KO mice compared with WT/Lox $(n=15)$

stimulation by glucose and $\mathrm{KCl}$ (Fig. 5a,b). To examine whether the effect of lipids on insulin secretion [37] was still intact in islets from beta HSL KO mice, we stimulated islets with $1 \mathrm{mmol} / \mathrm{l}$ palmitate in the presence of low or high glucose. Islets from both genotypes responded equally well to the combination of palmitate and glucose (Fig. 5c). Glucagon-like peptide-1 is an incretin that stimulates insulin secretion via a rise in cAMP and subsequent activation of PKA [38]. We therefore stimulated isolated islets with $100 \mathrm{nmol} / \mathrm{l}$ glucagon-like peptide-1; no differences were observed (Fig. 5d). Carbacholine potentiates insulin secretion via the phospholipase $\mathrm{C}$ pathway. Stimulation of insulin secretion with $100 \mu \mathrm{mol} / \mathrm{l}$ carbacholine was equally effective in islets from both genotypes (Fig. 5d). Finally, we stimulated islets with $\alpha$-ketoisocaproic acid $(\alpha-$ $\mathrm{KIC}$ ), which is known to exert strong insulinotropic effects; [38] stimulation of islets from beta HSL KO and WT/Lox mice by $10 \mathrm{mmol} / \mathrm{l} \alpha$-KIC did not reveal any significant differences in insulin secretion (Fig. 5d).

Capacitance and current recordings in single beta cells revealed a loss of the initial phase of insulin secretion in the absence of HSL Exocytosis evoked by a train of depolarisations resulted in a reduced exocytotic response in beta cells from KO mice (Fig. 6a,b). The total increase in membrane capacitance was reduced by $\sim 70 \%$ in beta cells from the beta HSL KO mice. Because exocytosis depends on the influx of $\mathrm{Ca}^{2+}$ through voltage-dependent calcium channels [39], we investigated the voltage-dependent $\mathrm{Ca}^{2+}$ currents. The maximum peak-current was similar in beta cells from both genotypes $(42 \pm 4(n=9)$ and $36 \pm 4 \mathrm{pA}(n=9)$, respectively). Thus, the reduction in exocytosis in the beta
HSL-KO mice is not due to a reduced influx of $\mathrm{Ca}^{2+}$ into beta cells.

Insulin content and beta cell mass are increased in beta HSL KO mice We found that insulin levels in islets from 12-week-old beta HSL KO mice were increased by 2.3-fold (Table 1). Using morphometrical analysis, we observed an increase in beta cell mass in beta HSL KO islets (Table 1). Thus, the impaired insulin secretory response cannot be attributed to a reduction in insulin content or beta cell mass. The observed increase in insulin content is likely to be accounted for by the increase in beta cell mass.

Plasma levels of adiponectin were unchanged in beta HSL KO mice, whereas whole-body fat and leptin levels were increased Adiponectin, and possibly leptin are known to enhance insulin sensitivity [40, 41]. Therefore, we determined adiponectin and leptin in plasma from 12-week-old mice. We found no significant differences in the plasma adiponectin levels (Table 1). However, a 3.5-fold increase in plasma leptin was observed in the KO mouse $(p<0.05$; Table 1). We therefore investigated body fat distribution in 12-week-old mice, and found a significant increase in overall fat content in beta HSL KO compared with WT/Lox mice (20 vs $15 \% ; p<0.01$; Table 1 ). Surprisingly, this increase in body fat did not affect total body mass since both WT/Lox and beta HSL KO mice displayed similar weight curves during the study (4 weeks WT/Lox 16.6 \pm 0.7 vs beta HSL KO $17.2 \pm 0.9 \mathrm{~g}[n=10] ; 12$ weeks WT/Lox $22.5 \pm 0.3$ vs beta HSL KO $22.8 \pm 0.9 \mathrm{~g}[n=10])$. The increase in body fat in the beta HSL KO mouse probably occurs at the expense of lean body mass. 

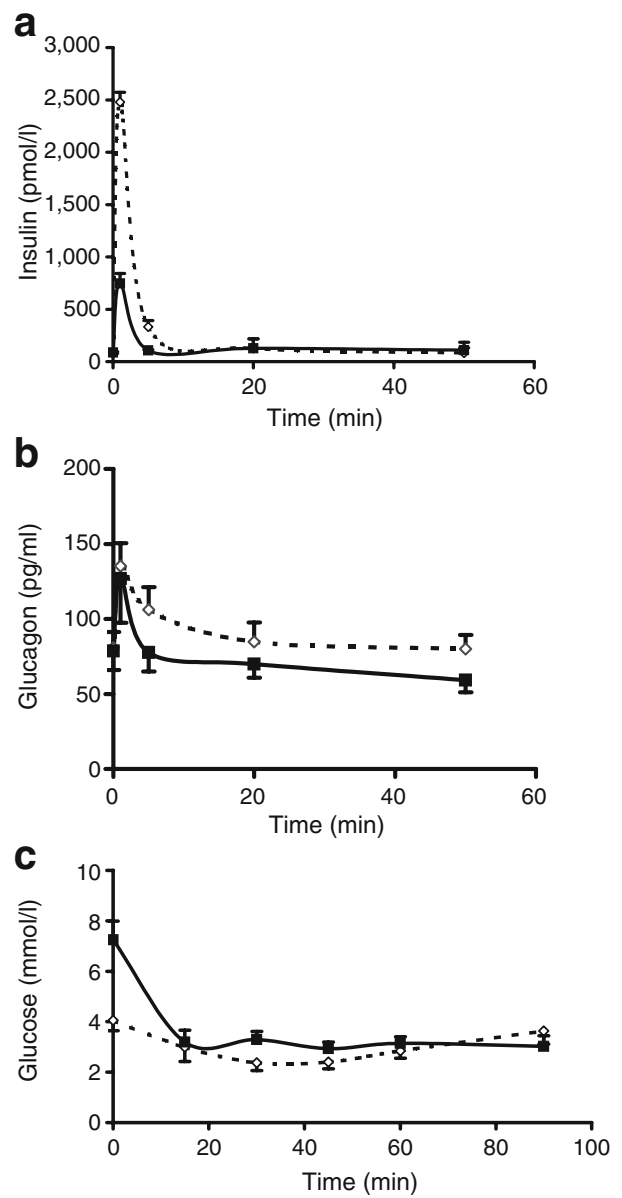

Fig. 3 Insulin release in response to arginine in an arginine tolerance test (ATT). a Insulin levels remain reduced in 12-week-old beta HSL KO (black squares) mice compared with WT/Lox (white diamonds)

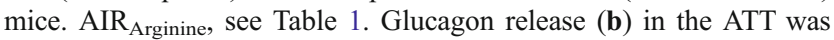
reduced in 12-week-old beta HSL KO islets (black squares) compared with WT/Lox (white diamonds) mice. AUC, see Table 1. c Differences in glucose clearance after an ITT in 10-week-old beta HSL KO (black squares) and WT/Lox (white diamonds) mice, respectively. For $\Delta$ values see Table 1 . Values are mean \pm SEM for two independent experiments; ten animals per group

Neutral lipids in islets An ablation of HSL could potentially cause an accumulation of triacylglycerol within the beta cells. We therefore stained pancreatic sections from both beta HSL KO $(n=4)$ and WT/Lox mice $(n=3)$ with Oil Red $\mathrm{O}$, which binds to neutral lipids. On visual inspection of islets in the sections, there were no apparent differences between the two genotypes (Fig. 7a,b). A quantitative analysis, assessing optical density, suggested a trend towards increased incorporation of lipid within the islets of beta HSL KO mice $(p=0.059)$.

For representative staining with Oil Red O, see ESM Fig. 2a, b, which shows staining of neutral lipids in islets from a normal-diet-fed C57BL/6J mouse compared with a high-fat diet-fed mouse.

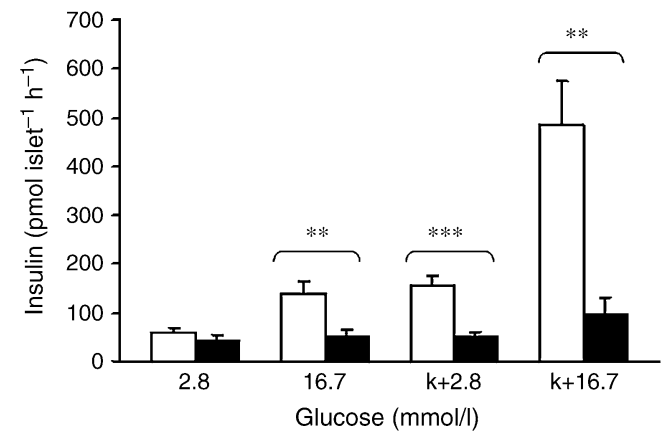

Fig. 4 Short (10 $\mathrm{min})$ static incubations in vitro. Islets from 9-weekold mice were incubated for $10 \mathrm{~min}$ with 2.8 and $16.7 \mathrm{mmol} / 1$ glucose in the presence of 4.8 or $70 \mathrm{mmol} / \mathrm{l} \mathrm{KCl}(\mathrm{k}+)$. Significant differences in insulin secretion were observed where beta HSL KO islets (black bars) failed to respond (vs WT/Lox, white bars). Values are means \pm SEM for three independent experiments using islets from two animals of each genotype. $* * p<0.01, * * * p<0.001$
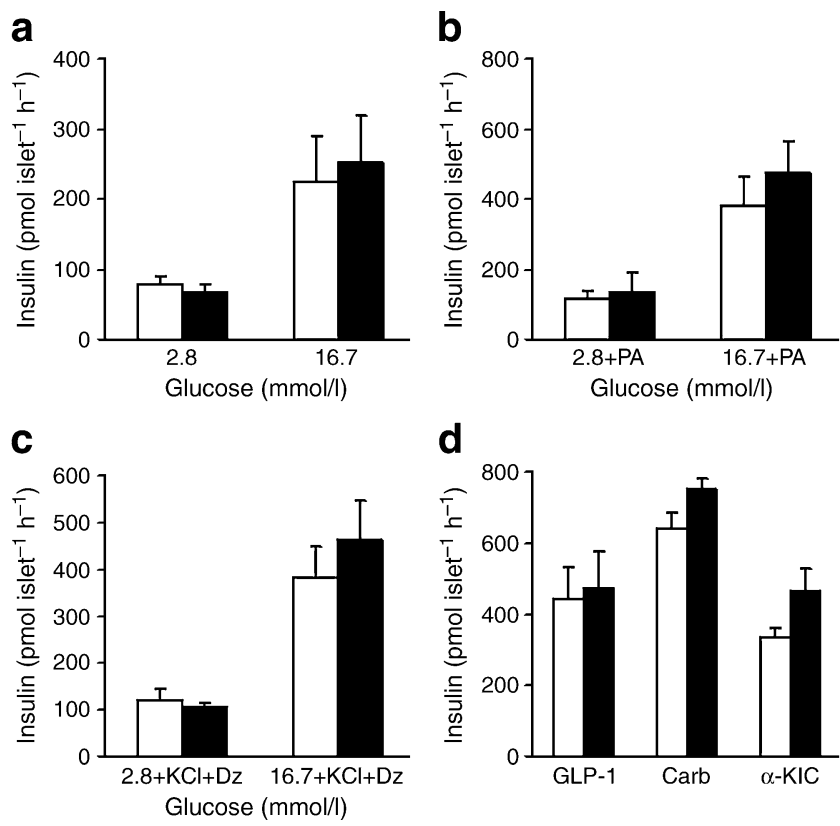

Fig. 5 Static $(1 \mathrm{~h})$ incubations in vitro. a Islets from 9-week-old beta HSL KO (black bars, all panels) and WT/Lox (white bars, all panels) mice were stimulated in vitro with 2.8 and $16.7 \mathrm{mmol} / 1$ glucose in $1 \mathrm{~h}$ batch incubation experiments. No significant differences were detected between the genotypes. Islets were then stimulated (b) with 2.8 and $16.7 \mathrm{mmol} / 1$ glucose in the presence of $35 \mathrm{mmol} / 1 \mathrm{KCl}$ and $250 \mu \mathrm{mol} / 1$ diazoxide (Dz; $\mathrm{K}_{\mathrm{ATP}}$-independent conditions). Here, too, no differences were detected. When stimulating islets (c) with $1 \mathrm{mmol} / 1$ palmitate (PA) at either 2.8 or $16.7 \mathrm{mmol} / 1$ glucose, islets from beta HSL KO mice responded equally well as islets from WT/Lox mice. d Islets were stimulated with $16.7 \mathrm{mmol} / \mathrm{l}$ glucose and $100 \mathrm{nmol} / 1$ glucagon-like peptide-1 (GLP-1) or $100 \mu \mathrm{mol} / 1$ carbacholine (Carb), or with $2.8 \mathrm{mmol} / 1$ glucose and $10 \mathrm{mmol} / 1 \alpha$-KIC. Again, no differences were seen. Values are means \pm SEM for three independent experiments using islets from two animals of each genotype 

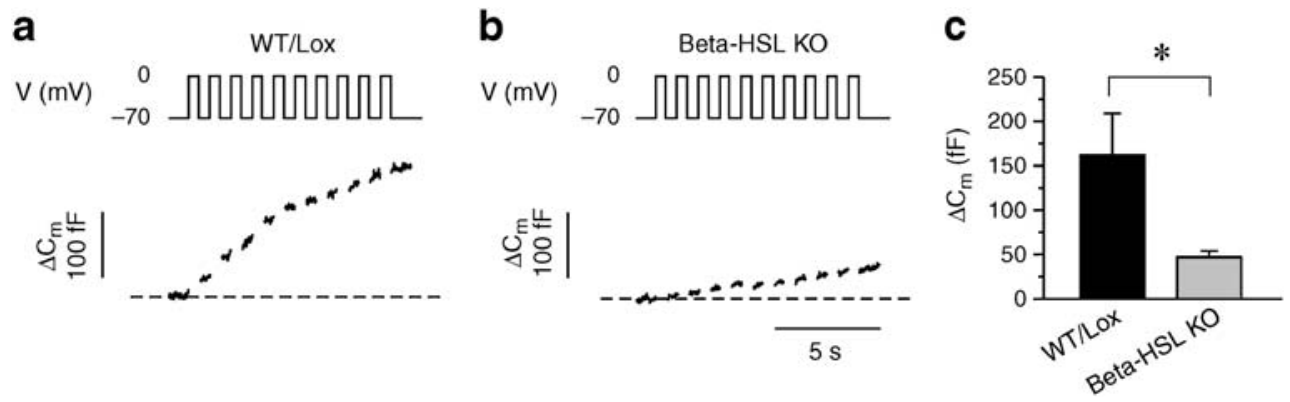

Fig. 6 Exocytotic response in the beta HSL-KO. Capacitance increase $\left(\Delta \mathrm{C}_{\mathrm{m}}\right)$ evoked by a train of ten $500 \mathrm{~ms}$ depolarisations from $-70 \mathrm{mV}$ to $0 \mathrm{mV}$ in a single beta cell from a WT/Lox mouse (a) and a beta HSL-KO mouse (b) as indicated. c Summary of the total increase in

Expression of Atgl is unaltered in islets from beta HSL KO mice Given the fact that ATGL has been discovered in adipose tissue and islets [29, 30] we investigated its expression in our model. Using quantitative real-time PCR, we found that Atgl was expressed in islets of our mice and thus could play a role in lipid metabolism in the beta cell. However, no significant difference in the mRNA expression of Atgl between beta HSL KO and WT/Lox islets was observed $(1.08 \pm 0.5$ vs $1.01 \pm 0.1 ; p>0.8 ; n=5$ for each group), suggesting that redundancy attributable to ATGL is unlikely in beta HSL KO mice.

\section{Discussion}

Following the discovery of HSL in pancreatic beta cells [5], several attempts to find a role for this lipase in the control of insulin secretion have been made. One view is that HSL could generate lipid signals that augment insulin secretion. For instance, LC-CoAs and diacylglycerol $[1,6]$ are both lipid-derived molecules that potentially could be generated

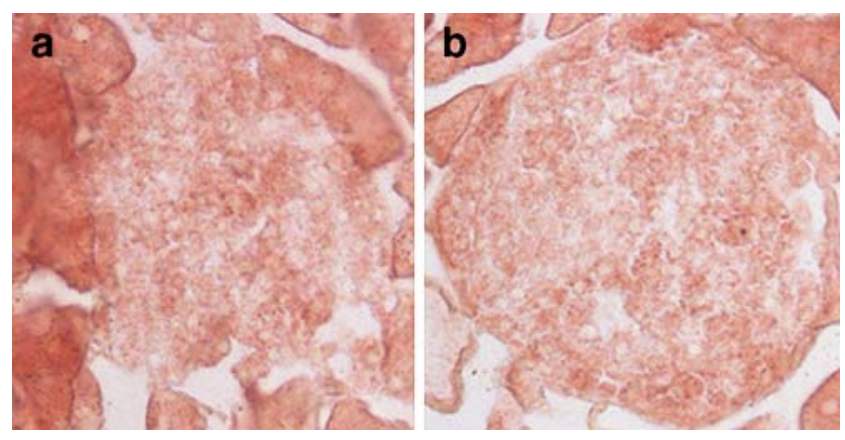

Fig. 7 Neutral lipid staining. Sections of pancreas and islets from 12week-old beta HSL KO (a) and WT/Lox mice (b), respectively were stained with Oil Red $\mathrm{O}$ for neutral lipids. The light pink colour has a similar intensity in both genotypes, indicating that there was no difference in lipid incorporation between the two membrane capacitance $\left(\Delta \mathrm{C}_{\mathrm{m}}\right)$ evoked by the train of depolarisations in single beta cells prepared from isolated islets from WT/Lox $(n=4)$ and beta HSL-KO $(n=5)$ mice. Data are mean values \pm SEM. ${ }^{*} p<0.05$

by HSL upon lipolysis. The role of lipases in insulin secretion has been emphasised by islet studies, using different pharmacological inhibitors [21, 42]. Recently, a specific inhibitor of HSL, 5-(2H)-isoxazolonyl urea, was shown to significantly inhibit insulin secretion in rat islets [14]. Together, these data suggest an important role for HSL in stimulus-secretion coupling and insulin secretion.

While pharmacological studies have supported the idea of a role for lipases in insulin secretion [13, 42], such studies have their shortcomings. It was therefore hoped that a global $\mathrm{KO}$ mouse would provide clear information about the role of HSL in beta cells. However, the different HSL KO lines exhibit varying phenotypes with respect to glucose homeostasis. Whereas the HSL KO mice created by Mitchell's laboratory exhibit both enhanced and impaired glucose-stimulated insulin secretion under different in vivo and in vitro conditions [27], the HSL-deficient mice created in our laboratory are insulin-resistant but compensate adequately with increased insulin secretion in vivo [21]. However, neither beta cell physiology nor insulin secretion has been studied in all global HSL KO lines [18, 20]. Therefore it is difficult to fully evaluate the observations on glucose homeostasis in these mouse lines. Nevertheless, the discrepancies may be attributed to differences in genetic background, genetic redundancy and/or the compensatory actions of other lipases. Thus, the lack of effect on insulin secretion in vitro in our global HSLdeficient mice [26] may be due to the compensatory actions of other lipases in islets. The identity of such (a) lipase(s) was until recently unknown. Previous work had suggested the action of another lipase in fibroblasts isolated from HSL-deficient mice [43]. Later, several groups identified a novel triacylglycerol lipase active in adipose tissue (ATGL) $[29,44]$. We have also found Atgl to be present in mouse islets and clonal beta cells [26]. This was further confirmed in the present study. Because Cre recombinase is not expressed until 11 days post conception [31], compensatory effects of other genes are less likely to occur. Therefore, a 
beta HSL KO could be less prone to redundancy and potentially clarify the role of HSL in beta cells.

In this study, we show that lack of HSL does indeed impact on insulin secretion. We showed that fasted beta HSL KO mice are hyperglycaemic and display retarded glucose clearance in vivo. The initial insulin secretory response in vivo and in vitro was blunted. This suggests that actions of HSL play an important role in the first phase of insulin release, possibly via a direct effect on exocytotic processes. We also showed that total insulin content was a 2.3-fold higher in beta HSL KO islets. Had we corrected for total insulin content in the in vitro secretion experiments, thus presenting the data as fractional insulin release, then not only early but also later phases of insulin secretion would appear as impaired. Recent work has shown that HSL is located in close proximity to the secretory granules in the pancreatic beta cell [45]. Our present findings therefore provide a functional correlate of this compartmentation of HSL. This is remarkably different from the function of HSL in adipocytes, where HSL is localised in the cytosolic compartment and translocates to the lipid droplet upon activation by PKA [4]. Whether such translocation occurs in beta cells is unknown. In fact, while very little is known about the structure of triacylglycerol stores in beta cells, studies have demonstrated lipid droplet formation within beta cells, either upon exposure to NEFA or in animals fed a high-fat diet $[16,35]$.

Changes in beta cell mass are observed in many mouse models of diabetes, emphasising the plasticity of beta cells in response to metabolic stress [46]. The changes in beta cell mass and total insulin content shown here are likely to be part of an intricate interplay of physiological and cellular mechanisms by which the beta cells attempt to maintain normal glucose levels. Because HSL is completely abolished from beta cells in our model, the increase of adipose tissue with the accompanying elevation of plasma leptin may also be part of these compensatory mechanisms to maintain glycaemic control. In fact, an increase in intraabdominal fat has been associated with insulin resistance, whereas an increase in subcutaneous fat correlates with increased leptin levels and improved insulin sensitivity [47]. While the DEXA scan performed on our beta HSL KO mice does not discriminate between different fat depots, visually the increase in adipose tissue appeared to be subcutaneous. This may explain the increase in leptin levels. Whether insulin sensitivity is affected in beta HSL KO mice remains unclear, since the data on insulin sensitivity were ambiguous. Clearly, an ITT does not take endogenous insulin secretion and the action of counterregulatory hormones into consideration.

Our study was not designed to examine the long-term effects of altered lipid metabolism on beta cells. However, because the ablation of HSL in theory could cause an accumulation of lipids within the beta cell, we nevertheless investigated islet lipid content, but were unable to detect any significant changes. This is consistent with our previous observations in the global HSL KO [26], but is in contrast to the findings in the Mitchell HSL KO line [27]. Using real-time PCR, we also measured mRNA levels of Atgl, a triacylglycerol lipase found to be active in adipocytes [29]. The lipase was expressed in mouse islets, but no differences in expression were found between the two genotypes, implying that compensation accounted for by ATGL, at least at the mRNA level, was not apparent in beta HSL KO islets.

Recently, concerns about glucose homeostasis in the RIP2 Cre mouse have emerged [48]. Lee et al. [48] reported that production of the CRE recombinase alone could be associated with glucose intolerance. This line is known to exhibit high levels of CRE recombinase in pancreatic beta cells, but also a low level in the hypothalamus [31]. It is a potential problem, since it has been proposed that HSL is produced in the hypothalamus [49]. However, we have been unable to detect any expression of Cre in the hypothalamus of our beta HSL KO mouse (data not shown). In addition, we have investigated glucose homeostasis and insulin secretion in vivo and in vitro in our line of RIP2 Cre mice [33]; no differences between transgenic and WT mice were found. We were thus unable to replicate previous findings [48]. The penetration of Cre expression in the brain may vary with different genetic backgrounds of the RIP2 Cre lines. Our RIP2 Cre line is thoroughly backbred on to a pure $\mathrm{C} 57 \mathrm{BL} / 6 \mathrm{~J}$ line, possibly explaining why it is less prone to genetic variability and also produces a clearer phenotype.

In conclusion, the data we present here suggest that HSL plays an important role in insulin secretion, possibly by producing lipid signals essential for the first phase of insulin release. The association of HSL with the secretory granules provides a structural correlate for these mechanisms [45]. Future work should focus on the identity of the lipid signals generated by HSL and perhaps other lipases in the beta cell.

Acknowledgements A.-H. Thorén Fischer and D. Persson provided technical assistance. Financial support was received from Swedish Research Council (14196-03A to H. Mulder, 13147 LE, 4499 to F. Sundler), NovoNordisk Foundation, Juvenile Diabetes Research Foundation, Albert Påhlsson, Åke Wiberg, Ingrid and Fredrik Thuring foundations, Swedish Diabetes Association, Swedish Medical Society, The Royal Swedish Physiographic Society, The Krapperup foundation (to M. Fex and F. Sundler), The Wilhelm Sander Stiftung (to M. Ristow), The Deutsche Forschungsgemeinschaft (to M. Ristow) and the Medical Faculty of Lund University.

Duality of interest The authors declare that there is no duality of interest associated with this manuscript. 


\section{References}

1. Prentki M, Corkey BE (1996) Are the beta-cell signaling molecules malonyl-CoA and cystolic long-chain acyl-CoA implicated in multiple tissue defects of obesity and NIDDM? Diabetes 45:273-283

2. Vaughan M, Berger JE, Steinberg D (1964) Hormone-sensitive lipase and monoglyceride lipase activities in adipose tissue. J Biol Chem 239:401-409

3. Yeaman SJ (2004) Hormone-sensitive lipase-new roles for an old enzyme. Biochem J 379:11-22

4. Holm C, Osterlund T, Laurell H, Contreras JA (2000) Molecular mechanisms regulating hormone-sensitive lipase and lipolysis. Annu Rev Nutr 20:365-393

5. Mulder H, Holst LS, Svensson H et al (1999) Hormone-sensitive lipase, the rate-limiting enzyme in triglyceride hydrolysis, is expressed and active in beta-cells. Diabetes 48:228-232

6. Corkey BE, Glennon MC, Chen KS, Deeney JT, Matschinsky FM, Prentki M (1989) A role for malonyl-CoA in glucosestimulated insulin secretion from clonal pancreatic beta-cells. J Biol Chem 264:21608-21612

7. Deeney JT, Gromada J, Hoy M et al (2000) Acute stimulation with long chain acyl-CoA enhances exocytosis in insulin-secreting cells (HIT T-15 and NMRI beta-cells). J Biol Chem 275:9363-9368

8. Hu L, Deeney JT, Nolan CJ et al (2005) Regulation of lipolytic activity by long-chain acyl-coenzyme $\mathrm{A}$ in islets and adipocytes. Am J Physiol Endocrinol Metab 289:E1085-E1092

9. Kanehara H, Suzuki J, Zenimaru Y et al (2004) Function of hormone-sensitive lipase in diacylglycerol-protein kinase $\mathrm{C}$ pathway. Diabetes Res Clin Pract 65:209-215

10. Yaney GC, Korchak HM, Corkey BE (2000) Long-chain acyl $\mathrm{CoA}$ regulation of protein kinase $\mathrm{C}$ and fatty acid potentiation of glucose-stimulated insulin secretion in clonal beta-cells. Endocrinology 141:1989-1998

11. Olofsson CS, Salehi A, Holm C, Rorsman P (2004) Palmitate increases L-type $\mathrm{Ca}^{2+}$ currents and the size of the readily releasable granule pool in mouse pancreatic beta-cells. J Physiol 557:935-948

12. Branstrom R, Leibiger IB, Leibiger B et al (2007) Single residue (K332A) substitution in Kir6.2 abolishes the stimulatory effect of long-chain acyl-CoA esters: indications for a long-chain acyl-CoA ester binding motif. Diabetologia 50:1670-1677

13. Mulder H, Yang S, Winzell MS, Holm C, Ahren B (2004) Inhibition of lipase activity and lipolysis in rat islets reduces insulin secretion. Diabetes 53:122-128

14. Claus TH, Lowe DB, Liang Y et al (2005) Specific inhibition of hormone-sensitive lipase improves lipid profile while reducing plasma glucose. J Pharmacol Exp Ther 315:1396-1402

15. Sorhede Winzell M, Ahren B (2004) Glucagon-like peptide-1 and islet lipolysis. Horm Metab Res 36:795-803

16. Cnop M, Hannaert JC, Hoorens A, Eizirik DL, Pipeleers DG (2001) Inverse relationship between cytotoxicity of free fatty acids in pancreatic islet cells and cellular triglyceride accumulation. Diabetes 50:1771-1777

17. Unger RH, Orci L (2000) Lipotoxic diseases of nonadipose tissues in obesity. Int J Obes Relat Metab Disord 24(Suppl 4):S28-S32

18. Osuga J, Ishibashi S, Oka T et al (2000) Targeted disruption of hormone-sensitive lipase results in male sterility and adipocyte hypertrophy, but not in obesity. Proc Natl Acad Sci U S A 97:787-792

19. Wang SP, Laurin N, Himms-Hagen J et al (2001) The adipose tissue phenotype of hormone-sensitive lipase deficiency in mice. Obes Res 9:119-128

20. Haemmerle G, Zimmermann R, Hayn M et al (2002) Hormonesensitive lipase deficiency in mice causes diglyceride accumulation in adipose tissue, muscle, and testis. J Biol Chem 277:4806-4815
21. Mulder H, Sorhede-Winzell M, Contreras JA et al (2003) Hormone-sensitive lipase null mice exhibit signs of impaired insulin sensitivity whereas insulin secretion is intact. J Biol Chem 278:36380-36388

22. Chung S, Wang SP, Pan L, Mitchell G, Trasler J, Hermo L (2001) Infertility and testicular defects in hormone-sensitive lipasedeficient mice. Endocrinology 142:4272-4281

23. Wang SP, Chung S, Soni K et al (2004) Expression of human hormone-sensitive lipase (HSL) in postmeiotic germ cells confers normal fertility to HSL-deficient mice. Endocrinology 145:5688 5693

24. Harada K, Shen WJ, Patel S et al (2003) Resistance to high-fat diet-induced obesity and altered expression of adipose-specific genes in HSL-deficient mice. Am J Physiol Endocrinol Metab 285:E1182-1195

25. Park SY, Kim HJ, Wang S et al (2005) Hormone-sensitive lipase knockout mice have increased hepatic insulin sensitivity and are protected from short-term diet-induced insulin resistance in skeletal muscle and heart. Am J Physiol Endocrinol Metab 289: E30-E39

26. Fex M, Olofsson CS, Fransson U et al (2004) Hormone-sensitive lipase deficiency in mouse islets abolishes neutral cholesterol ester hydrolase activity but leaves lipolysis, acylglycerides, fat oxidation, and insulin secretion intact. Endocrinology 145:3746-3753

27. Roduit R, Masiello P, Wang SP, Li H, Mitchell GA, Prentki M (2001) A role for hormone-sensitive lipase in glucose-stimulated insulin secretion: a study in hormone-sensitive lipase-deficient mice. Diabetes 50:1970-1975

28. Peyot ML, Nolan CJ, Soni K et al (2004) Hormone-sensitive lipase has a role in lipid signaling for insulin secretion but is nonessential for the incretin action of glucagon-like peptide 1 . Diabetes 53:1733-1742

29. Zimmermann R, Strauss JG, Haemmerle G et al (2004) Fat mobilization in adipose tissue is promoted by adipose triglyceride lipase. Science 306:1383-1386

30. Fex M, Lucas S, Sörhede Winzell M, Ahrén B, Holm C, Mulder $\mathrm{H}$ (2006) Beta-cell lipases and insulin secretion. Diabetes 55: S24-S31

31. Gannon M, Shiota C, Postic C, Wright CV, Magnuson M (2000) Analysis of the Cre-mediated recombination driven by rat insulin promoter in embryonic and adult mouse pancreas. Genesis 26:139142

32. Ristow M, Mulder H, Pomplun D et al (2003) Frataxin deficiency in pancreatic islets causes diabetes due to loss of beta cell mass. $\mathrm{J}$ Clin Invest 112:527-534

33. Fex M, Wierup N, Nitert MD, Ristow M, Mulder H (2007) Rat insulin promoter 2-Cre recombinase mice bred onto a pure C57BL/6J background exhibit unaltered glucose tolerance. J Endocrinol 194:551-555

34. Barg S, Galvanovskis J, Gopel SO, Rorsman P, Eliasson L (2000) Tight coupling between electrical activity and exocytosis in mouse glucagon-secreting alpha-cells. Diabetes 49:1500 1510

35. Fex M, Nitert MD, Wierup N, Sundler F, Ling C, Mulder $H$ (2007) Enhanced mitochondrial metabolism may account for the adaptation to insulin resistance in islets from $\mathrm{C} 57 \mathrm{BL} / 6 \mathrm{~J}$ mice fed a high-fat diet. Diabetologia 50:74-83

36. Brommage R (2003) Validation and calibration of DEXA body composition in mice. Am J Physiol Endocrinol Metab 285:E454 E459

37. Elks ML (1993) Chronic perifusion of rat islets with palmitate suppresses glucose-stimulated insulin release. Endocrinology 133:208-214

38. Kashima Y, Miki T, Shibasaki T et al (2001) Critical role of cAMP-GEFII-Rim2 complex in incretin-potentiated insulin secretion. J Biol Chem 276:46046-46053 
39. Ammala C, Eliasson L, Bokvist K, Larsson O, Ashcroft FM, Rorsman P (1993) Exocytosis elicited by action potentials and voltage-clamp calcium currents in individual mouse pancreatic Bcells. J Physiol 472:665-688

40. Ceddia RB, William WN Jr, Curi R (1999) Comparing effects of leptin and insulin on glucose metabolism in skeletal muscle: evidence for an effect of leptin on glucose uptake and decarboxylation. Int J Obes Relat Metab Disord 23:75-82

41. Yamauchi T, Kamon J, Waki $\mathrm{H}$ et al (2001) The fat-derived hormone adiponectin reverses insulin resistance associated with both lipoatrophy and obesity. Nat Med 7:941-946

42. Masiello P, Novelli M, Bombara M et al (2002) The antilipolytic agent 3,5-dimethylpyrazole inhibits insulin release in response to both nutrient secretagogues and cyclic adenosine monophosphate agonists in isolated rat islets. Metabolism 51:110-114

43. Okazaki H, Osuga J, Tamura Y et al (2002) Lipolysis in the absence of hormone-sensitive lipase: evidence for a common mechanism regulating distinct lipases. Diabetes 51:3368-3375

44. Villena JA, Roy S, Sarkadi-Nagy E, Kim KH, Sul HS (2004) Desnutrin, an adipocyte gene encoding a novel patatin domain- containing protein, is induced by fasting and glucocorticoids: ectopic expression of desnutrin increases triglyceride hydrolysis. J Biol Chem 279:47066-47075

45. Lindvall H, Nevsten P, Strom K et al (2004) A novel hormonesensitive lipase isoform expressed in pancreatic beta-cells. J Biol Chem 279:3828-3836

46. Masiello P (2006) Animal models of type 2 diabetes with reduced pancreatic beta-cell mass. Int J Biochem Cell Biol 38: 873-893

47. Cnop M, Landchild MJ, Vidal J et al (2002) The concurrent accumulation of intra-abdominal and subcutaneous fat explains the association between insulin resistance and plasma leptin concentrations : distinct metabolic effects of two fat compartments. Diabetes 51:1005-1015

48. Lee JY, Ristow M, Lin X, White MF, Magnuson MA, Hennighausen L (2006) RIP-Cre revisited, evidence for impairments of pancreatic beta-cell function. J Biol Chem 281:2649-2653

49. Sekiya M, Osuga J, Okazaki H et al (2004) Absence of hormonesensitive lipase inhibits obesity and adipogenesis in Lep ob/ob mice. J Biol Chem 279:15084-15090 